\title{
Valutazione visiva degli exit site nei cateteri venosi centrali in emodialisi: validazione preliminare di una scala italiana
}

\author{
Alessandro Carrus ${ }^{1}$, Elena Lovicu ${ }^{2}$, Dorian Soru ${ }^{1}$, Giovanni Chierroni ${ }^{1}$, Maria Grazia \\ Zunnui $^{1}$, Raffaella Maxia ${ }^{1}$, Francesco Logias $^{3}$ \\ ${ }^{1}$ Rete di Nefrologia e Dialisi, ASL di Nuoro, Nuoro \\ ${ }^{2}$ Infermiera, collaboratrice esterna alla ricerca, ASL di Nuoro, Nuoro \\ ${ }^{3}$ Direttore Rete di Nefrologia e Dialisi, ASL di Nuoro, Nuoro
}

\begin{abstract}
VISUAL EVALUATION OF THE CENTRAL VENOUS CATHETER'S EXIT SITE IN HEMODIALYSIS: PRELIMINARY VALIDATION OF AN ITALIAN SCALE Abstract. The central venous catheter (CVC) represents an alternative to the arteriovenous fistula (AVF) for patients on hemodialysis. However, CVCs are often cause of a large number of infective complications. Since nurses are the first healthcare professionals who detect possible infections, it is important to develop a visual scale to help their evaluation. This type of scale is important also for new nurses, as a way to take into consideration the main indicators of a possible CVC infection. Finally, this scale may be helpful to trace the evolution of a patient's CVC in his medical record. This study presents the first validation of an Italian scale that is used for the evaluation of CVC exit sites in hemodialysis. This scale is derived from the "Twardoski Scale". The validation involved the nurses of a Network of Nephrology and Dialysis, and an external nurse; the latter was not involved in the care of CVCs, and evaluated the exit site of the patients of the Network before the scale-aided evaluation by the nurse who took care of the CVC. The good Spearman correlation $(\rho=0.85)$ between the assessment of the external nurse and that of her colleagues confirms the goodness of the first Italian translation of the scale.
\end{abstract}

Key words: CVC scale in hemodialysis, Visual CVC scale, Exit site inflammation, Exit site care, Nursing

Conflict of interest: None.

Financial support: None.

Accettato: 26 Marzo 2014

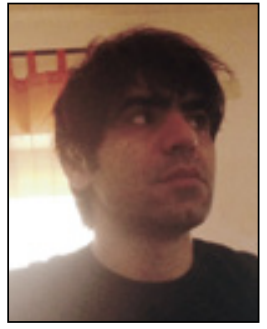

Alessandro Carrus

\section{Introduzione}

Il catetere venoso centrale (CVC) viene comunemente utilizzato in emodialisi, rappresentando frequentemente un'alternativa alla fistola arterovenosa (FAV). Il CVC viene definito come il "tallone d'Achille" dell'emodialisi (1), essendo spesso associato a numerose complicanze, specialmente di tipo infettivo (la presenza di un corpo estraneo nell'organismo rappresenta un substrato ideale per la colonizzazione microbica), ma anche meccaniche e sistemiche, comportando un aumento della morbidità e dei costi ospedalieri. La FAV rimane il gold standard per gli accessi vascolari nei pazienti in trattamento emodialitico. Secondo gli studi di Balwit e Rezabeck (2) rispetto alla FAV, il CVC presenta un tasso di complicanze molto più elevato e, più in particolare, Lacson e colleghi (3) evidenziano che il rischio di contrarre infezioni nei pazienti portatori di CVC è da $2 \mathrm{a}$ 9 volte superiore rispetto alla FAV e le cause di ricovero dei pazienti emodializzati per complicanze dell'accesso vascolare sono del $12.5 \%$.

L'infermiere è generalmente il primo operatore sanitario impegnato nella gestione e nell'osservazione del CVC; è, dunque, il primo a valutare i segni cardine dell'infiammazione: rubor, calor, tumor, dolor e functio lesa. È necessario che l'infermiere abbia a disposizione una scala di valutazione per l'identificazione e la valutazione visiva dei segni di infiammazione, in modo da uniformare i sistemi di valutazione dei singoli operatori. La scala rappresenta il trait d'union tra l'osservazione attuata dall'infermiere e gli esami di laboratorio con i quali si effettua la diagnosi. Una scala di questo tipo può essere 
uno strumento estremamente utile sia per rendere coerenti le valutazioni da parte del personale infermieristico sia per aiutare gli infermieri che si sono appena avviati alla professione a prendere in considerazione l'aspetto cruciale delle infezioni dei CVC. Rispetto al primo punto, accade, infatti, talvolta, che, in un'Unità Operativa, operino infermieri con esperienze diverse, i quali non necessariamente si sono occupati in maniera approfondita di emodialisi fino all'ingresso nell'Unità Operativa e che, conseguentemente, possono non avere immediatamente una visione condivisa di cosa sia potenzialmente un'infezione dell'exit site. Nel secondo caso, riguardante gli infermieri newcomer rispetto al mondo del lavoro, una scala condivisa e correttamente strutturata può essere un importante strumento di apprendimento. La scala può, inoltre, diventare parte integrante della cartella clinica del paziente, da utilizzare ogni volta che viene medicato il CVC. In questo modo, tutti gli operatori (anche quelli di un'altra Unità Operativa, in caso di trasferimento del paziente) hanno una visione completa della storia del CVC del paziente e possono intervenire con maggiori serenità e sicurezza.

In letteratura, vi sono molteplici articoli che descrivono, sotto numerosi punti di vista, il problema delle infezioni correlate al CVC (4-7), ma vi è un numero esiguo di lavori riguardanti una scala di valutazione visiva dell'infiammazione dell'exit site. Twardowski e Prowant (8) hanno elaborato una scala di valutazione del catetere nella dialisi peritoneale. Studi successivi hanno evidenziato la possibilità di utilizzare questa scala, apportando alcune modifiche, per la valutazione degli exit site dei $\operatorname{CVC}(9,10)$.

L'obiettivo del nostro studio è di costruire una prima versione italiana della scala modificata, a partire dall'elaborazione di Harwood et al. (9). Gli Autori, infatti, rendono disponibile la scala soltanto in lingua inglese e, perciò, si rendono necessarie una traduzione e una validazione per il contesto italiano. In questo studio, vengono presentati i risultati di una validazione preliminare e viene resa disponibile la scala così costruita per ulteriori studi. Questa prima validazione è, secondo noi, un passaggio importante per poter poi utilizzare la scala nell'Unità Operativa, fornendo agli operatori un valido strumento per un'analisi obiettiva e univoca del CVC.

\section{Materiali e metodi}

La validazione è stata effettuata in due fasi.

Nella prima fase, la scala, tradotta da noi in italiano (Fig. 1), è stata fatta utilizzare liberamente agli infermieri della Rete di Nefrologia e Dialisi dell'ASL di Nuoro, per un periodo di una settimana. Gli infermieri venivano invitati, subito dopo l'utilizzo, a riportare oralmente incomprensioni, imprecisioni od osservazioni rispetto alla scala stessa. Questa prima fase ci ha permesso di correggere la traduzione della scala in modo che fosse maggiormente comprensibile e condivisa. Abbiamo ripetuto ancora una volta questo passaggio, con le stesse modalità, in modo da verificare ulteriormente la comprensibilità della scala. Ciò ha permesso di costruire la versione utilizzata nella seconda fase (Fig. 2).
Nella seconda fase, durata una settimana, si è seguita la medesima procedura del lavoro di Harwood et al. precedentemente citato. Una delle Autrici del lavoro di adattamento linguistico (l'infermiera E. L.) ha valutato gli exit site dei CVC di tutti i pazienti della Rete di Nefrologia e Dialisi dell'ASL di Nuoro e, contestualmente, l'infermiere che effettuava la medicazione compilava la medesima scala. Per evitare di influenzarsi a vicenda, veniva chiesto agli infermieri, durante la medicazione dei CVC, di non effettuare alcun commento rispetto allo stato dell'exit site, a eccezione di quelli strettamente necessari per capire la presenza di problemi non visibili a occhio nudo (per esempio, informarsi dal paziente sulla presenza di prurito). Più in dettaglio, quindi, durante la medicazione, E. L. compilava la scala di valutazione e, subito dopo, la stessa scala veniva compilata dall'infermiere che aveva medicato il CVC. Questa fase ha, quindi, coinvolto $n=10$ pazienti $(7 \mathrm{~F}, 3 \mathrm{M})$, che hanno fornito il loro consenso alla partecipazione allo studio, per un totale di $n=12$ osservazioni (la differenza tra il numero dei pazienti e il numero di osservazioni è dovuta al fatto che, durante la settimana, alcuni CVC sono stati medicati più volte).

\section{Risultati}

Le valutazioni sono state codificate assegnando, per ogni item della scala, il punteggio 0 quando l'indicatore veniva valutato come assente e il punteggio 1 quando l'indicatore veniva valutato come presente. L'unica eccezione è rappresentata dall'item n. 8 ("Arrossamento nell' exit site"), in cui veniva chiesto ai valutatori di stimare anche la dimensione dell'arrossamento su una sottoscala a 6 livelli. In questo caso, è stato assegnato il punteggio 0 quando l'arrossamento veniva valutato come assente e il punteggio 1 quando l'arrossamento veniva valutato come $>3 \mathrm{~cm}$ (dimensione massima) e sono stati assegnati dei punteggi proporzionali (rispettivamente $0.167,0.333,0.500,0.667$ e 0.833 ) per gli altri livelli di valutazione. Si è, quindi, effettuata la somma della valutazione, ottenendo un valore compreso tra 0 (nessun indicatore presente) e 21 (tutti gli indicatori presenti, incluso l'arrossamento nell' exit site $>3 \mathrm{~cm}$ ).

I risultati sono stati analizzati utilizzando il software statistico gratuito e open source R (11). Dalle statistiche descrittive, elaborate grazie al pacchetto $R$ pastecs (12), si può evidenziare che il valore medio della somma nella valutazione di E. L. è pari a $2.278(\mathrm{SD}=1.445)$ mentre, per quanto concerne la valutazione degli altri infermieri, il valore medio è pari a $2.097(\mathrm{SD}=1.510)$. Questi risultati mostrano che, globalmente, i pazienti non presentano, da questa analisi, possibili infezioni importanti negli exit site dei CVC, come è auspicabile che accada per evitare le complicanze dovute all'uso del catetere. È mostrata di seguito (Fig. 3), elaborata grazie al pacchetto $R$ ggplot2 (13), la frequenza dei vari indicatori rilevati.

Si può evidenziare la presenza di arrossamenti della cute circostante l'exit site, che possono essere legati alla periodica necessità di rimuovere e riposizionare la medicazione. Nessun paziente riferisce dolore, ma sono numerosi quelli 
Azienda Sanitaria di Nuoro

Babass Baroses Mandrollis Maryine

Regione Autonoma della Sardegna

NUORO_03/01/2014

Direttore Generale Dott Antonio Maria Sory

Direttore Amministrativo Aw. Dort. Mario Giovanni, Atana

Direttore Sanitario Dott. Pieto Giovanni Mesina

RETE DI NEFROLOGIA E DIALISI DIRETTORE Dott. Francesco Logias Tel. $0784 / 240442$

neftrologianuoroa asinuoro it

RESPONSABLE INFERMIERISTICO Doth. Alessandro Carrus Tel. $0784 / 240343$

Data valutazione

Valutatore

$\square \quad$ II paziente sente dolore nell'exit site

\section{Oggetto: Modulo di valutazione degli exit site dei cateteri venosi centrali}

Per i seguenti indicatori: (apporre una $X$ se presente)

II paziente sente prurito nell'exit site

II paziente sente fastidio all'exit site

Febbre, se sì indicare la temperatura

La medicazione/benda si è staccata a casa

La cuffia è visibile

Trauma del lume (strattonato)

Arrossamento nell'exit site

Se presente stimare cortesemente la dimensione del margine dell'eritema attorno all'exit site

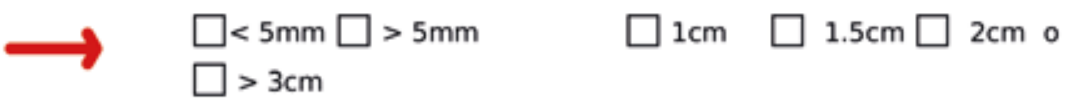

$\square$ Arrossamento lungo il decorso del catetere

$\square$ Gonfiore nell'exit site

$\square$ Gonfiore lungo il decorso del catetere

$\square$ Escoriazione asciutta nell'exit site*

$\square$ Crosta asciutta nell'exit site**

$\square$ Ascesso evidente (allargamento/indurimento sopra il tunnel)

$\square$ Essudato purulento nell'exit site (umido)

$\longrightarrow \square$ Essudato sieroso (umido)

$\square$ Essudato purulento che si presenta durante la manipolazione del catetere (umido)

$\longrightarrow \square$ Escoriazioni della pelle attorno all'exit site

$\square$ Tessuto di granulazione attorno all'exit site

Altro, specificare

II paziente presenta uno scarso livello di igiene? $\square$ Si $\quad \square$ No

* Escoriazione: definita come essudato indurito chiaro oppure scuro

** Crosta: definita come siero misto a sangue indurito wow.ashucrak drezionegenerale@asinuoro.it
Direzione Generale

Via Demurtas 108100 Nuoro

T. $078438892 / 240626$ F. 0784232095

Fig. 1 - Prima traduzione della scala con evidenziate le modifiche effettuate in seguito alla prima fase di validazione. 


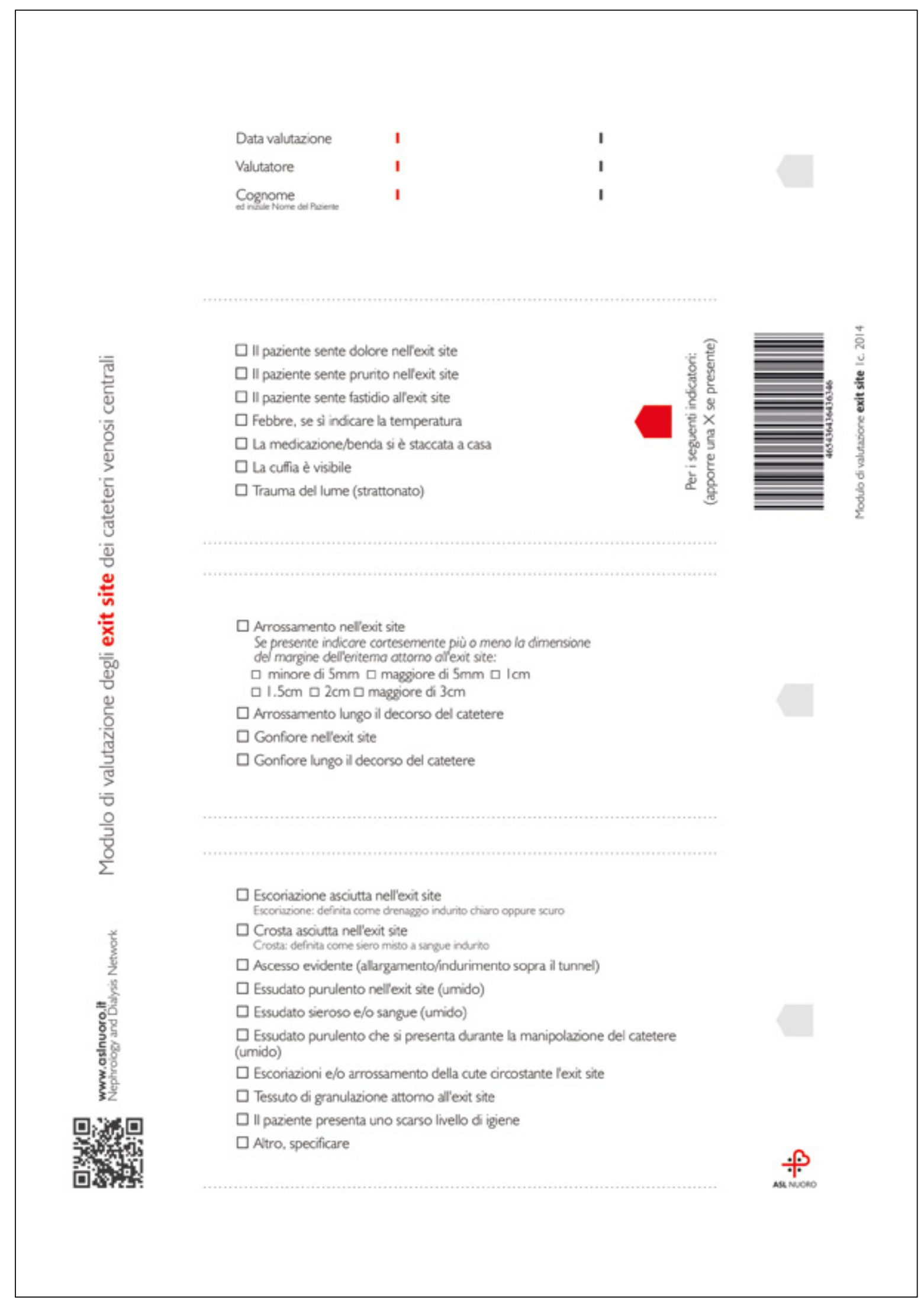

Fig. 2 - Scala utilizzata nella seconda fase. 


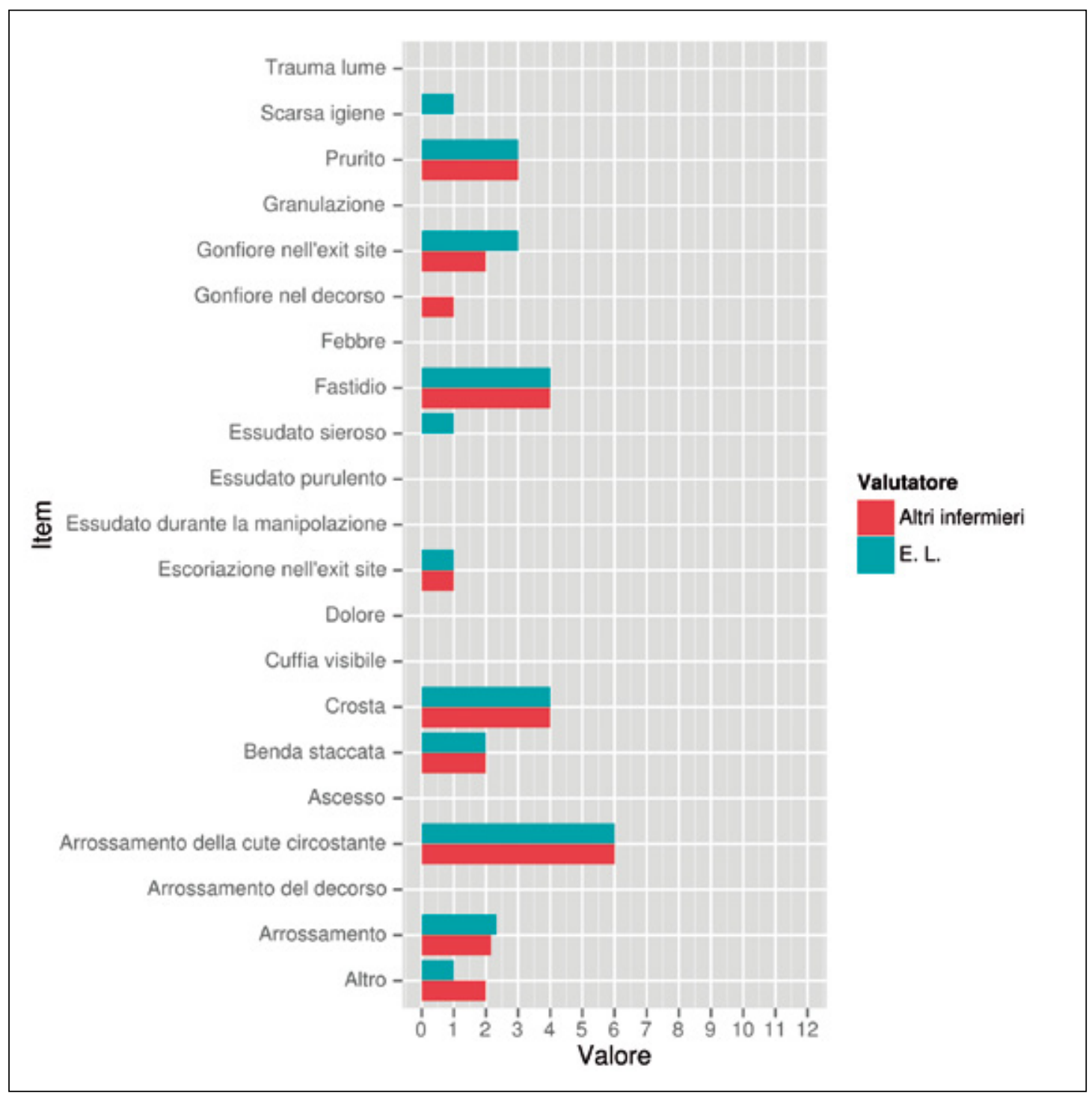

Fig. 3 - Frequenza degli indicatori della scala rilevati da $E$. L. e dagli altri infermieri.

che riferiscono fastidio e prurito, ma anche sollievo a seguito della medicazione. La crosta si può associare prevalentemente a cause meccaniche durante la medicazione. È stata, quindi, approfondita la presenza di una correlazione lineare tra la valutazione totale di E. L. e la valutazione totale degli altri infermieri. Dal momento che i punteggi totali non possono essere considerati una variabile continua, è stato applicato il test di correlazione per ranghi di Spearman. Preliminarmente è stata approfondita, graficamente, la presenza di una relazione monotonica tra i punteggi, poiché tale relazione è uno degli assunti della correlazione di Spearman. Come evidenziabile dal grafico sottostante (Fig. 4), elaborato tramite il pacchetto $R$ ggplot2 (13), in cui sono mostrate anche la retta di regressione e l'area corrispondente a un intervallo di confidenza del 95\% intorno alla retta di regressione, tale relazione è presente e appare essere una relazione monotonica crescente.
Si è, quindi, proceduto a calcolare la correlazione di Spearman tra il punteggio totale delle valutazioni di E. L. e il punteggio totale delle valutazioni degli altri infermieri. I risultati evidenziano una correlazione lineare positiva tra le due variabili: $\rho=0.85, p=0.0005$. Questo risultato è coerente con quello ottenuto da Harwood et al. (9), i quali hanno riscontrato un coefficiente di correlazione pari a $\rho=0.80$. Questo coefficiente di correlazione così elevato ci conferma di aver raggiunto l'obiettivo che ci eravamo posti: effettuare una validazione preliminare della scala per il contesto italiano.

\section{Conclusioni}

In questo lavoro, si è evidenziata inizialmente l'importanza di una scala visiva per la valutazione degli exit site dei CVC. Essa può essere uno strumento estremamente utile sia per fornire 


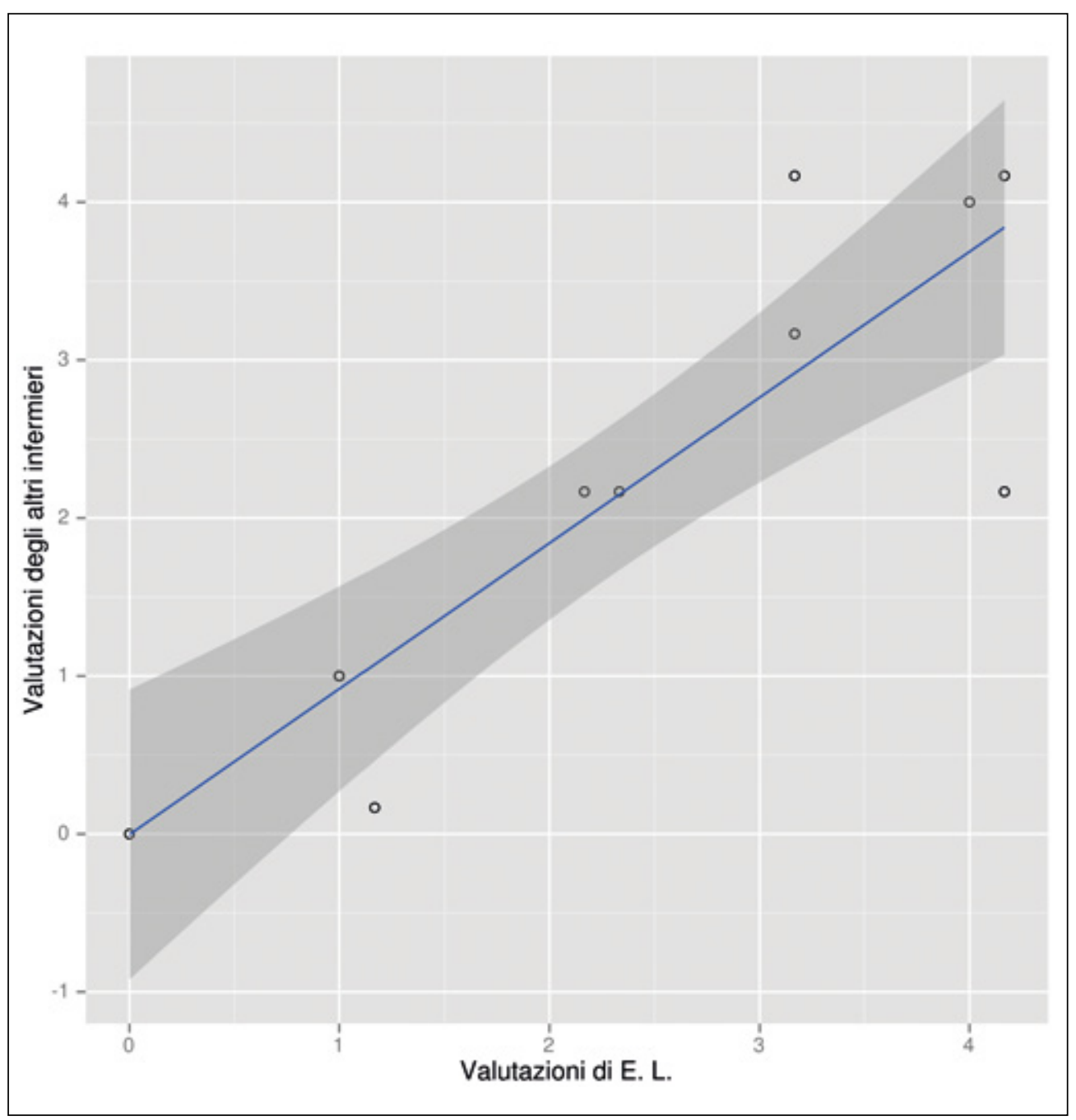

Fig. 4 - Grafico di dispersione delle valutazioni globali da parte di E. L. e degli altri infermieri.

una visione condivisa al personale infermieristico esperto, che lavora da tempo in un'Unità Operativa di Nefrologia e Dialisi sia per introdurre gli infermieri newcomer alla valutazione delle infezioni dei CVC e per prevenire, in tal modo, le complicanze ad esse associate. Rappresenta, inoltre, un importante strumento che, insieme alle analisi di laboratorio, permette di tenere traccia dell'evoluzione del catetere nella cartella clinica del paziente.

I risultati promettenti di questa prima validazione hanno consentito di sviluppare uno strumento che può essere sia ulteriormente validato, coinvolgendo un campione più ampio di pazienti, sia utilizzato in altri studi, per verificare l'efficacia di diverse medicazioni dei cateteri venosi centrali.

\section{Riassunto}

Il catetere venoso centrale (CVC) rappresenta, per i pazienti in emodialisi, un'alternativa alla fistola arterovenosa (FAV), ma, purtroppo, è spesso associato a un importante numero di complicanze infettive. L'infermiere è il primo operatore sanitario che rileva le possibili infezioni e, per questo, è importante sviluppare una scala visiva che gli sia di ausilio. Tale scala è importante anche per consentire a chi si avvicina da poco alla medicazione dei CVC di prendere in considerazione i principali indicatori di una possibile infezione. Essa, infine, può essere di ausilio per tenere conto dell'evoluzione del CVC nella cartella clinica del paziente. Questo studio presenta una prima validazione italiana di una scala utilizzabile per la valutazione degli exit site dei CVC in emodialisi (derivata dalla "Scala Twardowski"). La validazione ha coinvolto gli infermieri di una Rete di Nefrologia e Dialisi e un'infermiera esterna, non impegnata nella medicazione, la quale ha valutato gli exit site di tutti i pazienti della Rete, subito prima che la stessa scala venisse utilizzata da chi effettuava la medicazione. La buona correlazione di Spearman $(\rho=0.85)$ tra la valutazione dell'infermiera esterna e le valutazioni degli altri colleghi conferma la bontà di questa prima traduzione italiana della scala. 
Parole chiave: Scala CVC in emodialisi, Scala visiva CVC, Infiammazione degli exit site, Medicazione degli exit site, Assistenza infermieristica

Dichiarazione di conflitto di interessi: Gli Autori dichiarano di non avere conflitto di interesse.

Contributi economici agli Autori: Gli Autori dichiarano di non aver ricevuto sponsorizzazioni economiche per la preparazione dell'articolo.
Indirizzo degli Autori:

Dr. Alessandro Carrus

P.O. San Francesco

Via Mannironi 1

08100 Nuoro

alcarrus@gmail.com

\section{Bibliografia}

1. Lok CE, Mokrzycki MH. Prevention and Management of Catheter-related Infection in Hemodialysis Patients. Kidney Int 2011; 79 (6): 587-98.

2. Balwit JM: White Paper: Clinical and economic issues in vascular access for hemodialysis. Madison: Ahrens Balwit \& Association, Inc 2002.

3. Lacson E, Lazarus JM, Himmelfarb J, Ikizler TA, Hakim RL. Balancing fistula first with catheters last. Am J Kidney Dis. 20007; 50: 379-395.

4. Wagner J, Schilcher G, Zollner-Schwetz I, et al. Microbiological screening for earlier detection of central venous catheter-related bloodstream infections. Eur J Clin Invest 2013; 43 (9): 964-9.

5. Betjes MG. Prevention of catheter-related bloodstream infection in patients on hemodialysis. Nat Rev Nephrol 2011; 7 (5): 257-65.

6. Patel PR, Kallen AJ, Arduino MJ. Epidemiology, surveillance, and prevention of bloodstream infections in hemodialysis patients. Am J Kidney Dis 2010; 56 (3): 566-77.

7. De Andrade D, Ferreira V. Central venous access for haemodialysis: prospective evaluation of possible complications. J Clin
Nurs 2007; 16: 414-8.

8. Twardowski ZJ, Prowant BF. Exit-site study methods and results. Perit Dial Int 1996; 16 (Suppl. 3): S51-70.

9. Harwood L, Wilson B, Thompson B, Brown E, Young D. Predictors of Haemodialysis Central Venous Catheter Exit-Site Infections. The CANNT J 2008; 18 (2): 26-35.

10. Hughes K, Gardner A, McArdle J. Audit of factors associated with the intactness of central venous catheter exit site dressings for northern Australian haemodialysis patients. Ren Soc Aust J 2011; 7 (3): 108-14.

11. R Core Team. "R: a language and environment for statistical computing”. Vienna: R Foundation for Statistical Computing. Disponibile su http://cran.r-project.org/doc/manuals/r-release/ fullrefman.pdf (Accesso il 9 Marzo 2014).

12. Ibanez F, Grosjean P, Etienne M. Pastecs: package for analysis of space-time ecological series. Disponibile su http://cran.rproject.org/web/packages/pastecs/index.html (Accesso il 20 Dicembre 2013).

13. Wickam H. Ggplot2: elegant graphics for data analysis. New York: Springer 2009. 